\title{
POSSIBLE SOLUTIONS FOR INTERFERENCE COORDINATION IN HETNETS OF LTE-A
}

\author{
Koiloth SRS Jyothsna, T. Aravinda Babu, Joseph Michael Murray \\ Department of Electronics and Communication Engineering, \\ CBIT- Chaitanya Bharathi Institute of Technology, \\ Hyderabad, Telangana, India.
}

\begin{abstract}
In recent advances in cellular communication, the concept of heterogeneous networks has gained significant importance. A heterogeneous network is poised of multiple radio access technologies, architectures, transmission solutions, and base stations of varying transmission power that can interoperate, thus creating a multilayer structure. Management of interferences caused by the macro station to the low power nodes and vice versa is one of the biggest challenges in the deployment of heterogeneous networks. This paper presents a study of the mutual interference between a Macro cell and a Pico cell within LTE-A (Long Term Evolution - Advanced) framework. It is assumed that the Macro cell and the Pico cell share the same frequency channel and only the downlink $(D L)$ performance is studied. In this paper, the possible solutions of enhanced inter-cell interference coordination (eICIC) in terms of throughput and power are analyzed.
\end{abstract}

Key words: HetNet, intra frequency interference, interference coordination

Cite this Article: Koiloth SRS Jyothsna, T. Aravinda Babu, Joseph Michael Murray, Possible Solutions for Interference Coordination in HetNets of LTE-A, International Journal of Electrical Engineering and Technology (IJEET), 12(5), 2021, pp. 171-178. https://iaeme.com/Home/issue/IJEET?Volume $=12 \&$ Issue $=5$

\section{INTRODUCTION}

In 5G networks like LTE/LTE-A, however, inter-cell interference can be controlled through coordination among base stations. This was made possible because now LTE networks have $\mathrm{X} 2$ interfaces defined between base stations. By exchanging interference information over these $\mathrm{X} 2$ interfaces, base stations now can schedule radio resources in a way that avoids inter-cell interference.

There are several Interference Coordination technologies in LTE and LTE-A:

- LTE: Inter-Cell Interference Coordination (ICIC)

- LTE-A: Enhanced ICIC (eICIC) which is an adjusted version of ICIC for HetNet, and Coordinated Multi-Point (CoMP) which uses Channel Status Information (CSI) reported by UE 
Inter-Cell Interference Coordination (ICIC): The biggest cause of lower mobile network capacity is interference. Interference is caused when users in different neighbor cells attempt to use the same resource at the same time. Suppose there are two cells that use the same frequency channel and each cell has a UE that uses the same frequency resource.

As seen in the figure below, if the two UEs are located in cell centers like A2 and B2, no interference is caused because they use low power to communicate. However, if they are at cell edges like A1 and B1, their signals cause interference for each other because the two use high power to communicate.

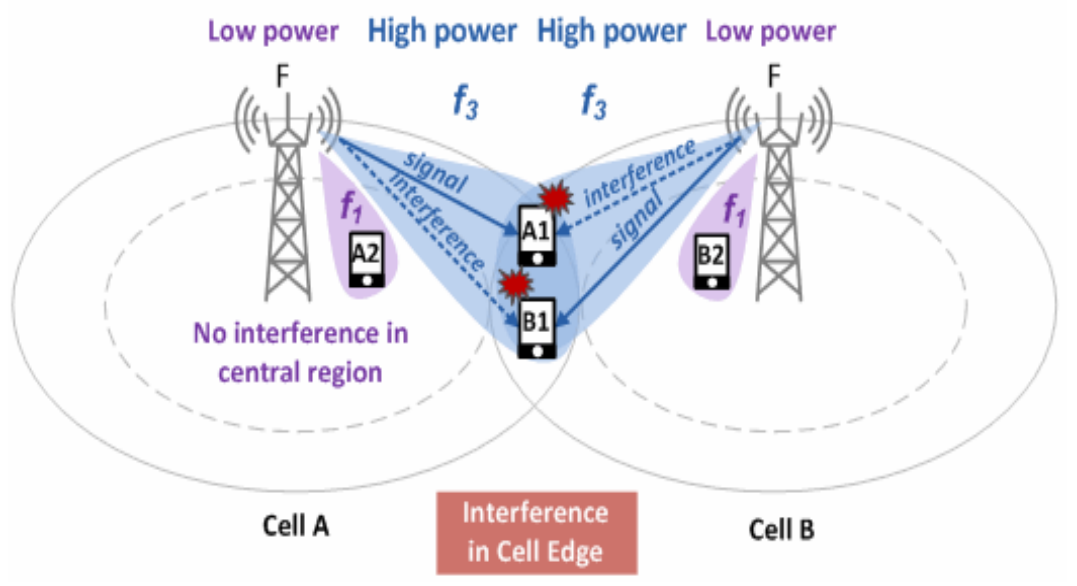

Figure 1 Typical scenario of interference with two UEs

\section{Concept of ICIC}

ICIC is defined in 3GPP release 8 as an interference coordination technology used in LTE systems. It reduces inter-cell interference by having UEs, at the same cell edge but belonging to different cells, use different frequency resources. Base stations that support this feature can generate interference information for each frequency resource (RB), and exchange the information with neighbor base stations through X2 messages. Then, from the messages, the neighbor stations can learn the interference status of their neighbors, and allocate radio resources (frequency, Tx power, etc.) to their UEs in a way that would avoid inter-cell interference.

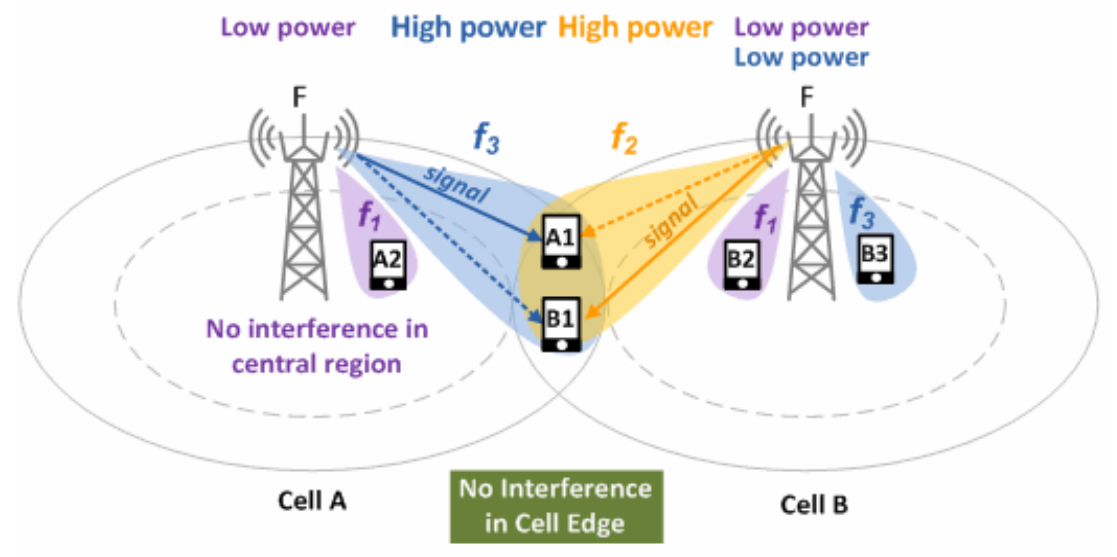

Figure 2 Scenario of ICIC with two UEs

In this approach, high interference indicator (HII), a bitmap, indicates scheduling of cell edge UEs. This message is exchanged between neighboring eNBs. The eNBs can thus coordinate their scheduling decisions and avoid interference for cell edge UEs. Additionally, 
an overload indicator (OI) indicates when cell edge UEs experience high interference. The receiving eNB is then able to adjust power and reduce interference.

\section{ENHANCED INTER-CELL INTERFERENCE COORDINATION (EICIC)}

Enhanced Inter-Cell Interference Coordination (eICIC), an interference control technology in LTE-A. The eICIC scheme focuses on the time domain solution to the interference problem. Networks consisting of the same type of cells are called homogeneous networks while ones with different types of cells are called heterogeneous networks (HetNet). So, HetNet is a network where small cells are deployed within a macro cell coverage.

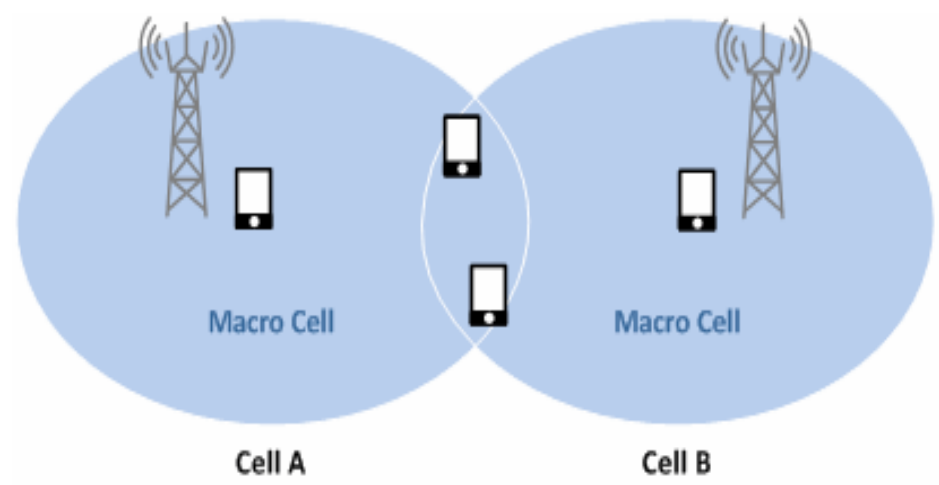

Figure 2 Homogenous Network

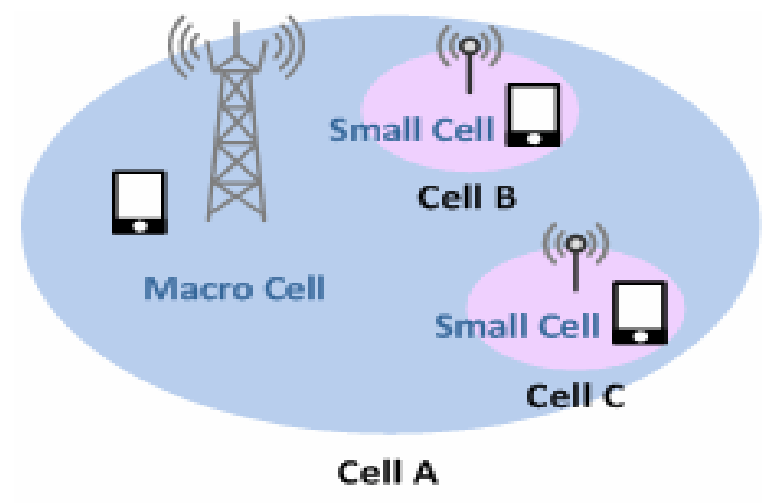

Figure 3 Heterogeneous Network (HetNet)

Concept of eICIC: It is an interference control technology defined in 3GPP release 10. It is an advanced version of ICIC, previously defined in 3GPP release 8, evolved to support HetNet environments. To prevent inter-cell interference, ICIC allows cell-edge UEs in neighbor cells to use different frequency ranges (RBs or sub-carriers). On the other hand, eICIC allows them to use different time ranges (subframes) for the same purpose. That is, with eICIC, a macro cell and small cells that share a co-channel can use radio resources in different time ranges (i.e. subframes).

Two main features of eICIC are: Almost Blank Subframe (ABS) technology defined in Release 10 and Cell Range Expansion (CRE) technology defined in Release 11. ABS can prevent cell-edge UEs in small cells from being interfered with by the neighboring macro cell by having both cells still use the same radio resources, but in different time ranges (subframes). CRE expands the coverage of a small cell so that more UEs near cell edge can access the small cell. In this post, we will discuss ABS only. 

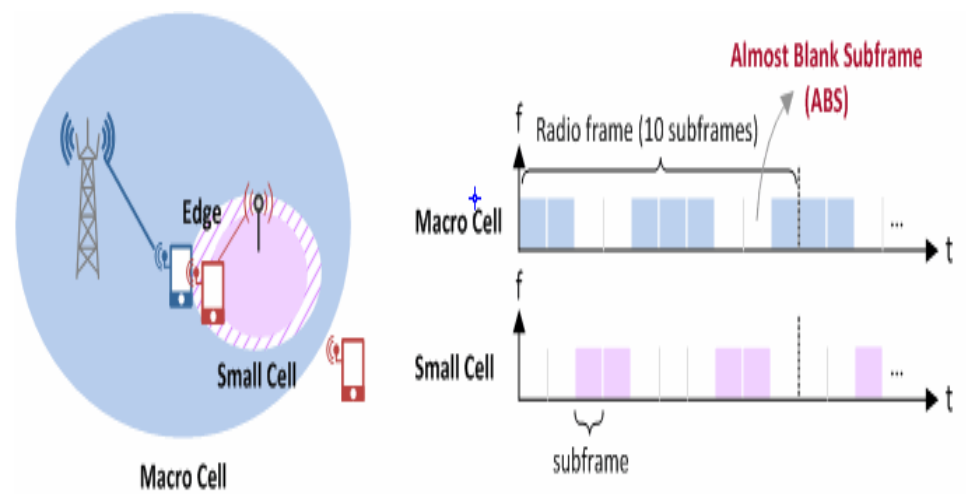

Figure 4 Concept of ABS in HetNets

ICIC enables cell-edge UEs to use different frequency resources (RBs) in communicating, by having neighboring base stations exchange interference information with each other over $\mathrm{X} 2$ interface. This is effective in reducing inter-cell interference in an existing macro cell-based homogeneous network, but causes interference between control channels in a HetNet.

When a base station communicates with a UE, each DL subframe of $1 \mathrm{msec}$ consists of two periods - one for delivering control channel and the other for delivering data channel. ICIC can allocates different frequency resources to cell-edge UEs only when delivering data channels (Physical Downlink Shared Channel; PDSCH). Resource information allocated to UEs is delivered through control channels (Physical Downlink Control Channel; PDCCH). Here the thing is, unlike data channels, control channels are not delivered through different frequency ranges, but distributed across the entire channel bandwidth first and then delivered. This may cause UEs in neighbor cells to share the same frequency resources.

\section{POSSIBLE SOLUTIONS FOR EICIC}

Recently, numerous techniques have been presented in the literature to control downlink transmission power of the femtocell. A distributed joint resource allocation algorithm has been proposed to mitigate ICI in the two-tier femto-macro network. This algorithm consists of spectrum sensing, transmission mode selection and channel state information (CSI) estimation.

However, all the techniques in the literature are power control based. Hence, in this paper, all the possible solutions to control interference in HetNets is presented.

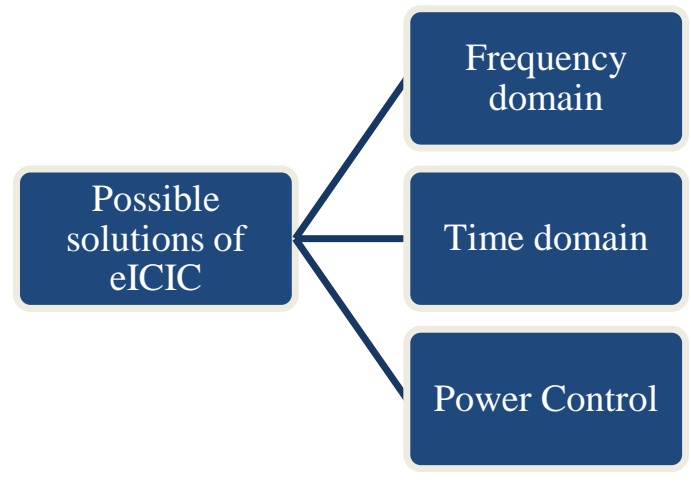

Figure 5 Possible solutions of eICIC

i. Frequency domain solution: In Frequency Domain, one way to reduce the interference would be to allocate the resource blocks from multiple neighboring cells in such a way that the allocated resource blocks does not overlap each other. For example, if a cell (e.g, serving cell) allocated RB0 10 for a UE, let neighboring cell allocate RB 15 20 for another UE. 
ii. Time domain solution: In Time Domain, one way to reduce the interference is that a cell (e.g, serving cell) stop transmitting at a certain subframe so that other cell (e.g, a femto/pico cell) can transmit the signal during that period. But sometimes completely stopping the signal transmission would cause some issues. So it would be recommended to transmit the signal in very low power instead of completely stopping the transmission. This subframe with very low signal power is called 'Almost Blank Subframe (ABS).

iii. Power control Solution: In Power control mechanism, HeNB adjust power to avoid interference to other nodes. This is a technique where the HeNB transmit power is reduced to minimize the interference to macro UEs while still providing sufficient performance to the HeNB UEs. This autonomous power calibration method is based on measuring the signal strength from the strongest co-channel deployed macro eNB, followed by adjusting the HeNB transmit power as a linear function of this measurement.

Active power control technique (APC): The downlink transmission power of femtocell causes interference to the MUE that existing in its coverage area. In such condition, MUE starts the handover or cell re-selection process. Since the femtocell is working in closed access mode, the macro user cannot subscribe to the services of the femtocell.

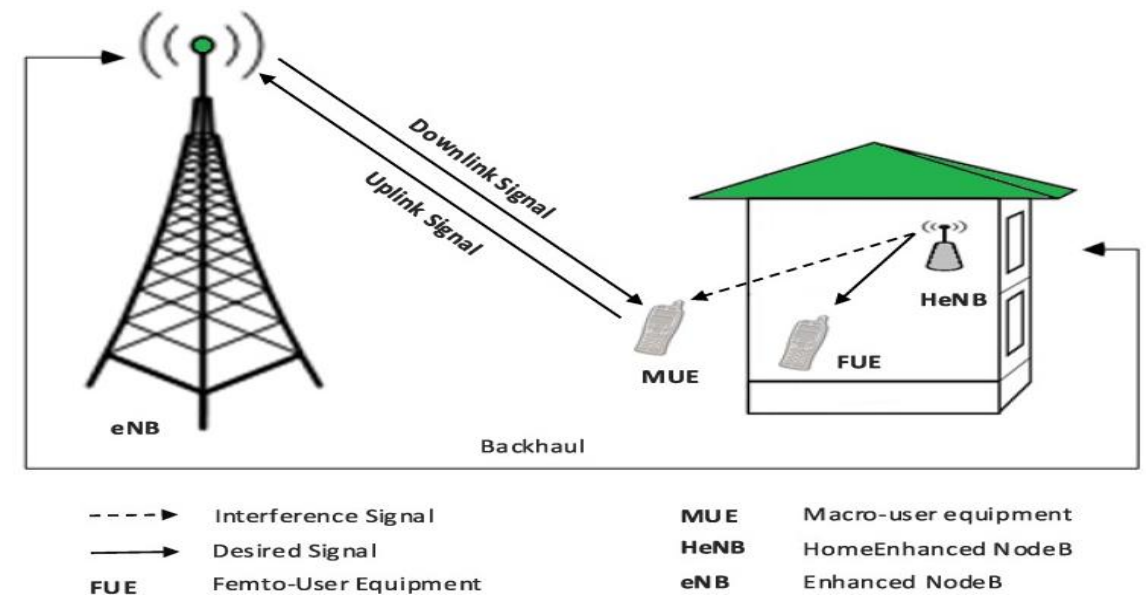

Figure 6: Interference scenario in HeNB and nearby macro and Femto users

In order to deal with such a scenario, the MUE measures the interference level from the HeNB and sends an interference message (IM) to the femtocell based on the Interference Indication Function (IDF). The IDF determines whether the interference experienced by the macro user is higher or lower than the threshold interference level.

Performance comparison of active power control technique with the conventional techniques:

- The Active Power Control Technique effectively reduces the inter-cell interference and optimize the throughput performance of the UE.

- This technique not only reduces ICI to UE but also maintains the QoS by considering the RSRP (Reference Signal Received Power) feedback from the Femto user to adjust its downlink transmission power.

- The femtocell actively tunes its downlink power by using the power levels and time levels. Hence, this approach reduce the unnecessary power consumption to achieve green femtocell network.

- Compared with existing power control approaches, this approach offers significantly better performance in terms of downlink throughput CDF of the macro user and the femto user, the average throughput, FBS Power consumption and the green impact and $\mathrm{CO} 2$ emission. 
Among all the possible solutions discussed in this section, LTE-A uses time domain solution for cancelling the interference.

\section{COMPARATIVE ANALYSIS OF ALL POSSIBLE SOLUTIONS}

For interference cancellation in LTE-A, the following conditions are required:

\section{i. At the base station:}

- Multi Input Multi Output (MIMO): All the stream parameters should be known.

- Intracell: All the stream parameters should be known, joint multi user detection can be carried out.

- Intercell: All the stream parameters should be known, blind detection of interference.

\section{ii. At the terminal:}

- Multi Input Multi Output (MIMO): All the stream parameters should be known.

- Intracell and Intercell: All the stream parameters should be known, needs blind detection or extra signaling.

In LTE-A, eICIC employs intra carrier management technique for HetNets. In macro-Pico HetNets, the conventional ABS subframe in macro cells is almost muted except for some necessary control information, and it is denoted as zero-power ABS (ZP-ABS). However, this will decrease the spectral efficiency of macro cells with ZP-ABS subframes. Power control can be applied to ABS subframes, which are called low-power ABS (LP-ABS) in macro cells to allow BSs to serve UEs with good channel condition using a reduced transmission power, as long as the low transmission power in macrocell ABSs would not incur a high ICI to picocells. Current eICIC is mainly designed for cross-tier ICI coordination with a dominant interferer in different layers.

\section{RESULTS AND DISCUSSION}

In this paper, interference coordination in HetNets is analyzed with all possible solutions of eICIC. Among the possible solutions, time domain and power control are highly optimal. In the following analysis, the proposed eICIC is considered to be the power control technique i.e. active power control (APC) technique.

The technique is implemented within and outside HetNets so that the improvement of throughput can be clearly shown. The improvement in throughput will obviously control the interference in macro-Pico HetNets.

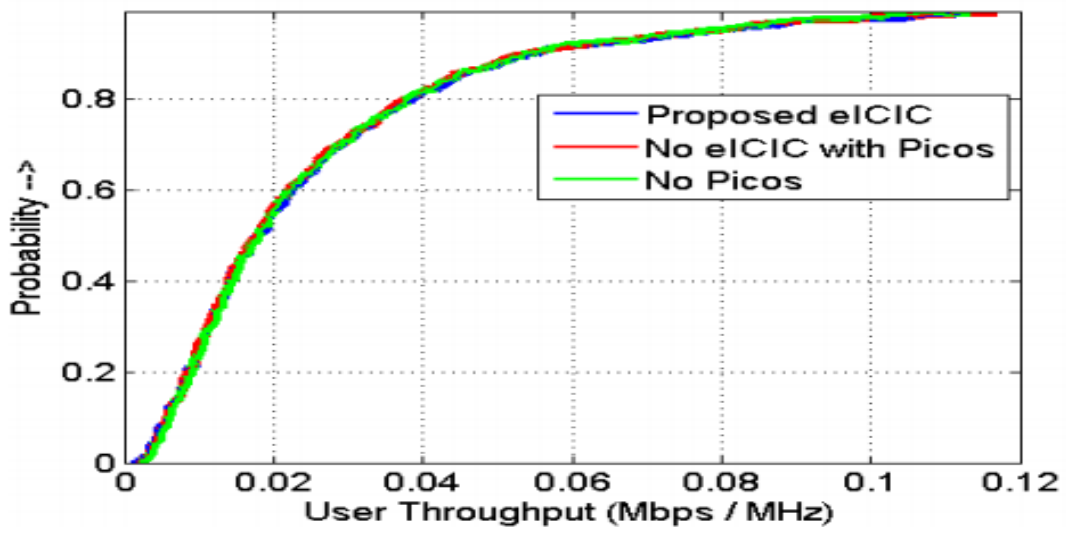

Figure 7: Throughput improvement using eICIC outside macro-Pico HetNets 
From the above plot, it is obvious that eICIC gives almost same performance outside the macro-Pico HetNets in terms of throughput. In this analysis, the proposed eICIC technique considered is active power control (APC).

As the main aim of this paper is to control the interference within macro-Pico HetNets, the proposed techniques in implemented within the HetNets. The simulation results are encouraging and are shown below:

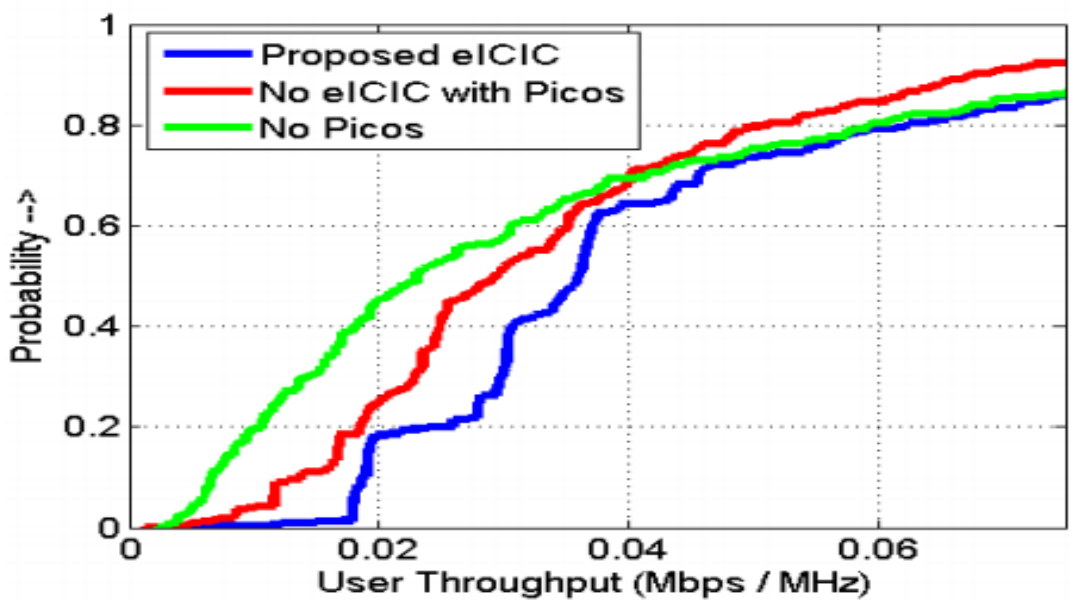

Figure 8: Throughput improvement using eICIC inside macro-Pico HetNets

The above plot is the analysis within HetNets. It is very much clear that, the proposed eICIC has improved the throughput in macro-Pico HetNets.

\section{CONCLUSION}

This paper presented possible solutions for interference mitigation or coordination. A detailed description of frequency domain, time-domain and power control solution of e-ICIC is presented. eICIC for macro-femto and macro-pico HetNets from the perspective of standardization and advanced optimized designs is well explained. It has shown that there is always a dominant interferer in HetNets, resulting in severe cross-tier ICI, and eICIC is an effective technique to reduce it by subframe coordination. Finally, from both the theoretical analysis and the perspective of 5G and beyond, the open issues of eICIC have been discussed. It is observed that, active power control (APC) technique offers significantly better performance in terms of throughput and power consumption.

\section{REFERENCES}

[1] Xing C, Gao F, Zhou Y. A Framework for Transceiver Designs for Multi-Hop Communications With Covariance Shaping Constraints. IEEE Trans Signal Process, 2015, 63: 3930-3945.

[2] Zhang Z, Long K, Wang J. Self-organization paradigms and optimization approaches for cognitive radio technologies: a survey. IEEE Wirel Commun, 2013, 20: 36-42.

[3] K.S.R.S. Jyothsna, T.Aravinda Babu, "Interference Mitigation Using E-Comp in Multi-Layered Networks of LTE”, IJEEE, Vol 8, issue 01, January - June 2016.

[4] Jun Z., Hui T., Peng T.,Y.H., Liqi G., 2012.Dynamic Frequency Reservation Scheme for Interference Coordination in LTE-Advanced Heterogeneous Networks, IEEE Vehicular Technology Conference (VTC Spring), pp.1-5, doi: 10.1109/VETECS.2012.6239915.

[5] 3GPP (2012) Evolved Universal Terrestrial Radio access (E-UTRA) and Evolved Universal Terrestrial Radio Access (E-UTRAN), overall description 3GPP TS v Third Generation Partnership Project, Technical Report. 
[6] G. Foder et al., "Intercell Interference Coordination in OFDMA Networks and in 3GPP Long Term Evolution Systems,” IEEE J. Commun., vol. 4, no. 7, Aug. 2009, pp 445-53.

[7] A. Khandekar et al., "LTE-Advanced: Heterogenous Net-works," IEEE Proc. European Wireless Conf., Apr. 2010, pp 987-82.

[8] G. Boudreau et al., "Interference Coordination and Can-cellation for 4G Networks," IEEE Commun. Mag., Apr. 2009, pp. 74-80.

[9] Erik Dahlman, Stefan Parkvall, Johan Skold,. "4G, LTE-Advanced Pro and the Road to 5G". London, United Kingdom: Academic Press. 2016.

[10] Hitachi (2011) R, System Design Considerations for CoMP and eICIC. Technical Report, August 2011. 